\title{
La maternité des déesses grecques et les déesses- mères : entre mythe, rite et fantasme
}

Vinciane PIRENNE-DELFORGE

\section{(2) OpenEdition \\ 1 Journals}

Édition électronique

URL : https://journals.openedition.org/clio/1452

DOI : $10.4000 /$ clio. 1452

ISSN : 1777-5299

Éditeur

Belin

Édition imprimée

Date de publication : 1 avril 2005

Pagination : 129-138

ISBN : 2-85816-781-8

ISSN : 1252-7017

Référence électronique

Vinciane PIRENNE-DELFORGE, "La maternité des déesses grecques et les déesses-mères : entre mythe, rite et fantasme », Clio. Histoire, femmes et sociétés [En ligne], 21 | 2005, mis en ligne le 01 juin 2007, consulté le 22 avril 2022. URL : http://journals.openedition.org/clio/1452 ; DOI : https://doi.org/ $10.4000 /$ clio. 1452

Ce document a été généré automatiquement le 22 avril 2022.

Tous droits réservés 


\title{
La maternité des déesses grecques et les déesses-mères : entre mythe, rite et fantasme
}

\author{
Vinciane PIRENNE-DELFORGE
}

1 La notion de maternité recèle au moins deux dimensions : la capacité reproductive des femmes, d'une part, les soins apportés à leur progéniture, de l'autre. Pour évoquer un monde divin conçu sur le modèle de la société humaine, les Grecs ont abondamment utilisé le schéma généalogique. C'est une des articulations de leurs discours sur les dieux et le monde. La capacité reproductive des dieux est donc pensée sur le mode de la génération humaine et les exceptions à cette règle, comme les parthénogenèses, n'invalident pas la pertinence du modèle général. En outre, l'union sexuelle qui met en jeu une divinité aboutit forcément à une naissance puisque les dieux sont infiniment féconds. Pourtant, la virginité se rencontre parmi les déesses. Pour trois d'entre elles, le statut de parthenos est explicitement revendiqué au début du grand Hymne homérique à Aphrodite: Athéna, Artémis et Hestia refusent de se soumettre à la loi universelle de l'union sexuelle. Un tel choix les exclut a priori de la sphère des mères, du moins sur le plan biologique. Mais la «biologie » n'est pas tout. La maternité est aussi métaphore, l'image de la sollicitude, de la générosité et du souci pour ses propres enfants ou pour d'autres figures qui requièrent une protection. Les deux dimensions de la maternité ne sont donc pas toujours associées chez les déesses. Deux exemples seront ici envisagés pour l'illustrer : tout d'abord, celui des «mères » de la Théogonie d'Hésiode, ensuite, le cas un peu particulier d'une Athéna « mère » située à Élis.

Les mères de la Théogonie d'Hésiode

2 Dans la Théogonie d'Hésiode, trois déesses portent le titre de "mère »: Gaia, la terre présente dès l'aube du cosmos, Rheia, l'une de ses filles, et Déméter, l'une de ses petites-filles, qui manifeste le mot mètèr dans son nom même. Le cas de Gaia est riche de sens. Elle est la puissante génitrice primordiale qui inaugure l'ordre généalogique de la construction du monde : seule, puis unie au Ciel, elle enfante les composantes de ce monde (v. 126-153). Toutefois, le terme de mètèr ne deviendra explicite qu'avec le 
premier épisode dramatique de la cosmogonie. En effet, le Ciel refuse de laisser venir ses enfants au jour en maintenant sa partenaire dans une copulation permanente. La Terre fait alors appel à ses enfants pour la délivrer - et leur permettre de voir le jour dans le même mouvement. C'est à ce moment précis qu'elle devient explicitement la "mère ", dans la réponse positive que Cronos lui adresse : «... le grand Cronos aux idées retorses, trouva soudain quel langage tenir en réplique à sa noble mère : 'Mère, peut-être que ce que tu dis - oui je m'y engage - je pourrais l'accomplir...' » (v. 168-170, trad. A. Bonnafé). L'épisode offre la première image circonstanciée d'une relation entre la mère et ses enfants, au-delà du simple processus générateur : comme si le nom même de mère recelait forcément une dimension relationnelle plus constitutive de ce statut que la seule reproduction. Au vers 284, c'est une des composantes de Gaia, à savoir le chthôn, qui est qualifié de "mère des troupeaux». Le chthôn est la partie grasse et fertile de la terre, l'humus qui permet la croissance de la végétation. En l'occurrence, l'image de la maternité circonscrit le caractère nourricier de la terre: c'est donc davantage la sollicitude et le soin que la génération proprement dite qui sont ici impliqués.

3 Cronos renouvelle ensuite le crime paternel et avale ses enfants «sitôt que chacun, quittant les entrailles sacrées de sa mère, arrivait à ses genoux » (v. 459-460). La parturition de Rheia justifie biologiquement le titre que le poète lui attribue. Mais ce n'est pas tout. La dramatisation de l'épisode lui accorde, à ce stade de la théogonie, le même rôle que celui de Gaia à la génération précédente: elle dissimule Zeus à la voracité de Cronos et lui donne une pierre emmaillotée à la place de son fils. On connaît la suite : Zeus devenu grand fera rendre raison à Cronos, qui vomira ses enfants dans la foulée. La sollicitude de Rheia, la mère, double son rôle de génitrice. Elle autorise le déblocage de la théogonie et, à terme, la mise en place de la souveraineté de Zeus.

$\mathrm{Au}$ terme du processus théogonique, Zeus s'unit à des déesses auxquelles il donne des enfants. Une seule d'entre elles, pourtant, reçoit explicitement le nom de mère : c'est Déméter qui «enfanta Perséphone aux bras blancs qu'Aidôneus enleva d'auprès de sa mère et se vit remettre en don par Zeus maître de l'idée » (v. 912-914). La maternité de Déméter apparaît dans la deuxième partie de son nom, mais le récit de l'enlèvement de sa fille dramatise ce statut de mère et donne à son nom une consistance remarquable. Quant à la première partie du nom, Dè-, elle pose des problèmes d'interprétation ${ }^{1}$, mais que le préfixe fasse référence à la terre ou au grain, la dimension de «nourricière » de la déesse est d'emblée soulignée par l'épithète poluphorbè que lui attribue la Théogonie (v. 912).

5 Selon un principe bien mis en lumière par J. Rudhardt, le processus théogonique spécialise dans les jeunes dieux les potentialités de leurs parents² ${ }^{2}$. Les correspondances entre Gaia, Rheia et Déméter relèvent d'un tel principe : toutes trois sont liées à la fertilité de la terre, mais quand les déesses primordiales assument cette qualité sur un plan presque générique, Déméter est tout particulièrement liée à l'agriculture et donc à un stade plus spécialisé de l'alimentation humaine. Toutes trois sont des "mères »; néanmoins, dans les cités et leurs sanctuaires, la dramatisation de la maternité telle que l'évoque la Théogonie - Gaia et la castration d'Ouranos, Rheia et le vomissement de ses enfants, Déméter et le deuil de celle qui devient Perséphone - est principalement associée à ce couple "mère-fille ". L'Hymne homérique à Déméter raconte ce drame tellement connu qu'Hésiode le résume en deux vers. Soulignons simplement combien, dans cette superbe évocation hymnique, le thème de la mère en colère s'ajuste 
complètement à celui de la terre stérile. Le deuil de la mère divine fait courir un danger de mort aux humains, mais il ne pose pas moins problème au monde divin puisque le subtil équilibre qui s'est instauré entre la protection accordée par les dieux et les sacrifices offerts par les hommes est rompu.

Le cadre festif des Thesmophories s'articule au récit fondateur de l'enlèvement de Korè, du deuil de sa mère et de son retour auprès d'elle ${ }^{3}$. Or les Thesmophories sont une des fêtes grecques les plus anciennement attestées et les plus largement répandues dans tout le monde grec $^{4}$. La fête dramatise rituellement le souci de la communauté, à la fois pour sa subsistance matérielle - la fertilité de son territoire -, et pour sa continuité - la fécondité de ses femmes. Le réseau symbolique de la maternité de Déméter, que ce soit dans le mythe ou dans le culte, associe étroitement les deux lieux de la pérennité des sociétés. Ce profil de nourricière et de mère sous-tend également la création par Déméter de ses mystères à Éleusis, censés donner aux hommes de douces espérances pour l'au-delàs. La sollicitude divine dont une telle fondation témoigne émane du deuil et de la douleur d'une mère et prend en charge la faiblesse constitutive de l'humanité : sa mortalité. C'est donc en tant qu'individu mortel - et non en tant que citoyen ou en tant que membre de tel ou tel cercle de sociabilité - que l'initié aux mystères se met sous la protection maternelle de la déesse.

\section{L'Athéna mètèr d'Élis}

7 Athéna est une de ces déesses qui gardent jalousement leur virginité, avec Artémis et Hestia, on l'a dit. Comment comprendre, dès lors, qu'un récit étiologique de la cité d'Élis lui attribue le titre de Mètèr? L'histoire se passe en ces temps anciens de la venue d'Héraclès en Élide pour nettoyer les écuries d'Augias. Le roi refuse de payer son prestigieux ouvrier pour son intervention et le héros ravage la région avant de s'apaiser à la suite d'un oracle. Pausanias, qui rapporte ce récit au II siècle de notre ère, enchaîne en affirmant que "les femmes d'Élide, comme leur pays était privé de population adulte, adressèrent, dit-on, des prières à Athéna pour être enceintes dès qu'elles auraient des relations avec leurs hommes; leur prière fut exaucée et elles fondèrent un sanctuaire à Athéna surnommée Mère. En outre comme aussi bien les femmes que les hommes avaient retiré un plaisir particulier à ces unions, à l'endroit où ils s'étaient unis pour la première fois, ils appellent cet endroit 'Délicieux' et le fleuve qui coule là, ils l'appellent aussi 'Eau délicieuse' $»^{6}$.

8 Au moment de visiter la cité d'Élis et ses environs, Pausanias ne parle plus de cette singulière qualification. Sur l'acropole des Éléens, rapporte-t-il, se trouve un sanctuaire d'Athéna dont la statue d'or et d'ivoire, qui porte un coq sur son casque, est attribuée à Phidias. L'oiseau a une nature combative, poursuit-il, mais pourrait être tout autant consacré à Athéna Erganè (« l'Artisane »)7. Il n'est donc pas question ici d'une Athéna Mètèr et le récit qui la concerne, rapporté au milieu de l'historique de la région, semble détaché de tout contexte cultuel, du moins au temps de la visite de Pausanias. Il en va de même pour un autre sanctuaire d'Athéna. C'est dans un récit étiologique que Pausanias raconte sa fondation par Narkaios, le fils de Dionysos et d'une certaine Physkoa à qui est destiné un chœur à Olympie. Une telle consécration honore la déesse en la qualifiant de Narkaia pour avoir favorisé les victoires guerrières et la puissance de Narkaios ${ }^{8}$. Mais de sanctuaire réel, croisé au cours de la visite, il n'est jamais question.

Ce petit dossier autour de la maternité d'Athéna est exemplatif, non seulement des difficultés de comprendre la logique " polythéiste » pour nous qui sommes étrangers au système en question, mais aussi de la richesse du champ sémantique que recouvre le 
terme grec de mètèr en contexte divin. Dans l'application stricte des fonctionnalités divines au sein du panthéon "panhellénique » et au vu de la mention explicite du plaisir sexuel, on s'attendrait à trouver une Aphrodite dans la prière des Éléennes, en tant que déesse qui favorise la pulsion érotique. ${ }^{9}$ À défaut d'une Aphrodite, on se contenterait bien d'une Artémis, censée protéger les parturientes et leurs bébés. Mais non : c'est Athéna que supplient les femmes en mal d'homme et d'enfant. Comment comprendre cette étrangeté ? Pour cela, le détour par Athènes s'impose puisque Nicole Loraux nous a naguère montré que là vivaient Les enfants d'Athéna.... ${ }^{10}$. Or, à Athènes, la «maternité » d'Athéna est un motif travaillé par les mythes fondateurs. L'histoire est connue. Poursuivie par les assiduités d'Héphaïstos, la déesse esquive une éjaculation qui produit son fruit dans la terre sous les espèces du petit Érichthonios. Athéna prend alors le bébé en charge et le dissimule dans une corbeille laissée à la garde des filles du roi, les Kékropides. Curieuses, elles soulèvent le couvercle. Le spectacle interdit les frappe de folie et elles se suicident du haut de l'Acropole.

est donc la mère "biologique " de l'enfant, mais Athéna s'affirme comme la mère de tous les Athéniens : « Souveraine (potnia), ce sol est à toi, et cette cité : tu es sa mère (matèr) et sa maitresse (despoina) et sa gardienne (phulax) ", disent les vieillards de Marathon mis en scène par Euripide ${ }^{11}$. Cette maternité symbolique est réaffirmée chaque année dans le rite. Deux petites filles reçoivent de la prêtresse d'Athéna un colis mystérieux dont même la prêtresse ignore le contenu. Elles empruntent un escalier raide et souterrain au cœur même de la citadelle pour émerger non loin du sanctuaire de l'Aphrodite des Jardins au flanc nord de l'acropole. Elles laissent là ce qu'elles ont apporté et repartent avec un autre colis enveloppé. On ignore le déroulement exact de la cérémonie, mais les petites arrhéphores - tel est leur nom - quittent alors le service de la déesse qui siège sur l'acropole et sont remplacées par d'autres ${ }^{12}$. Que ces fillettes rejouent le drame des Kékropides a été reconnu depuis longtemps ${ }^{13}$. Que le colis fasse d'une manière ou d'une autre référence au petit Érichthonios est une hypothèse de poids. Comme l'écrivait J. Rudhardt, l'action des arrhéphores « assure constamment la naissance de nouveaux Athéniens $»^{14}$. Athéna patronne ainsi la survie de la communauté, qu'elle protège comme une mère, et le rituel contribue à assurer, sans solution de continuité, la pérennité de la cité, avec la collaboration - indéterminée - de l'Aphrodite des Jardins.

11 Munis de ces arguments, revenons à Élis. La tradition y est bien moins riche et Pausanias est notre seul informateur. Néanmoins, les analogies entre les deux traditions sont frappantes : l'Athéna d'Élis est acropolitaine | celle d'Athènes l'est aussi ; l'Athéna Narkaios est liée à la puissance guerrière d'un souverain | l'Athéna d'Athènes est liée aux rois du lieu; l'Athéna Mètèr intervient dans un contexte guerrier et de crise qui menace la survie même de la communauté | l'Athéna "nourrice» d'Érichthonios assure elle aussi la pérennité de sa cité ; la puissance génératrice liée au fleuve " délicieux » est associée à la pulsion sexuelle et au plaisir érotique | l'Aphrodite des Jardins est associée au rituel des arrhéphories athéniennes. Ajoutons que, dans le texte de Pausanias, c'est Phidias a réalisé pour l'Athéna d'Élis une statue chryséléphantine (or et ivoire), entrant donc en résonance avec le chef-d'œuvre que le sculpteur athénien avait donné à sa cité sous la forme de l'Athéna Parthénos !

Le raisonnement par analogie n'est pas dépourvu de danger. Toutefois, le faisceau de convergences ainsi dégagé est suffisamment fourni pour espérer éviter la surinterprétation. L'Athéna Mètèr et l'Athéna Narkaia des récits étiologiques recueillis à 
Élis par Pausanias ${ }^{15}$ sont des variations mythiques sur le thème de "l'Athéna qui protège sa cité ", à l'instar de l'Athéna acropolitaine dont les enfants sont Athéniens. Il ne faut sans doute pas chercher la trace de ces sanctuaires ailleurs que sur l'acropole d'Élis, là où s'élevait la statue de Phidias. Mais, comme souvent, le détail du travail de réélaboration de ces traditions éléennes nous échappe.

L'Athéna Mètèr relève donc d'une maternité métaphorique, répondant à une logique polythéiste complexe, dont les cultes de Déméter nous ont eux aussi montré la richesse.

Restent les fantasmes annoncés par le titre. En bref. Toutes les déesses que l'on vient d'évoquer dans leur puissance maternelle et de décliner selon les lieux symboliques qui les voient apparaître (théogonie et hymne poétique, étiologies locales, rituels civiques) ont été, à un moment ou à un autre, identifiées comme autant d'avatars d'une puissance maternelle primordiale. Même Athéna, dont on a montré le rapport complexe à la maternité, n'a pas échappé à la règle ${ }^{16}$. Des réactions constructives ont vu le jour, montrant combien cette fascination des origines et le fantasme quasi psychanalytique d'une "Déesse-Terre-Mère " étaient redevables d'une lecture historiquement déterminée des sources héritée $d u x X^{e}$ siècle $^{17}$. Certains courants féministes ont à leur tour sacrifié, en l'infléchissant, au mythe d'un "monothéisme féminin ancestral $»^{18}$. Ce sont évidemment des cas extrêmes de surinterprétation des données. Il n'en reste pas moins qu'une forme de paresse langagière et de négligence conceptuelle contribue régulièrement à voir resurgir les notions floues de "Déessemère » ou de "Terre-mère » en contexte grec. Les quelques exemples envisagés dans le cadre de cette brève contribution montrent combien de telles étiquettes, sans être totalement inadéquates, doivent être nuancées en fonction des contextes où se voit énoncée une qualité de "mère " pour toutes ces déesses. De telles catégories, qu'on le veuille ou non, sont chargées du poids de toute une tradition historiographique. Elles figent artificiellement des profils divins auxquels la souplesse des panthéons locaux confère en réalité un grand dynamisme. Elles tendent à l'unification inappropriée d'une expérience religieuse qui est plurielle et complexe.

\section{BIBLIOGRAPHIE}

BRULÉ Pierre, 1987, La fille d'Athènes, Paris.

BURKERT Walter, 1966, «Kekropidensage und Arrhephoria », Hermes 94, p. 1-25 (trad. franç. dans

Sauvages origines. Mythes et rites sacrificiels en Grèce ancienne, Paris, 1998 [Vérité des

mythes], p. 71-111).

CALAME Claude, 1997, «L'Hymne homérique à Déméter comme offrande », Kernos 10, p. 111-133.

DONNAY Guy, 1997, « L'arrhéphorie : initiation ou rite civique ? », Kernos 10, p. 177-205.

FOLEY Helen (éd.), 1994, The Homeric "Hymn to Demeter", Princeton.

FRAZER R.M., 1969, « Some Notes on the Athenian Entry, Iliad B 546-556 », Hermes 97, p. 262-266. 
GEORGOUDI Stella, 1991, « Bachofen, le matriarcat et le monde antique. Réflexion sur la création d'un mythe ", in in G. Duby, M. Perrot (éds), Histoire des femmes. 1. L'Antiquité, Paris, p. 477-491. --, 2002, « Gaia/Gê. Entre mythe, culte et idéologie », in S. Des Bouvrie (éd.), Myth and Symbol I, Bergen, p. 113-134.

GOODISON Lucy, MORRIS Christine, 1998, Ancient Goddesses. The Myths and the Evidence, British Museum Press.

HAARMANN Harald, 1996, Die Madonna und ihre griechischen Töchter. Rekontruktion einer kulturhistorischen Genealogie, Hildesheim.

LORAUX Nicole, $1990^{2}$ [1981], Les enfants d'Athéna. Idées athéniennes sur la citoyenneté et la division des sexes, Paris.

--, 1990, Les Mères en deuil, Paris.

--, 1991, «Qu'est-ce qu'une déesse ? », in G. Duby, M. Perrot (éds), Histoire des femmes. 1.

L'Antiquité, Paris, p. 31-62.

MOTTE André, 1986a, « Mort et renaissance dans les mystères d'Éleusis », in Mort et fécondité dans les mythologies. Actes du colloque de Poitiers (mai 1983), Paris, p. 71-82.

--, 1986b, « Silence et secret dans les Mystères d'Éleusis », in Les rites d'initiation. Actes du colloque de Liège et de Louvain-la-Neuve (novembre 1984), Louvain-la-Neuve.

PIRENNE-DELFORGE Vinciane, 1994, Kernos, supplément 4 : L'Aphrodite grecque, Liège.

--, 2004, « Qui est la Kourotrophos athénienne ? ", in V. Dasen (éd.), Naissance et petite enfance dans l'Antiquité. Actes du colloque de Fribourg (novembre-décembre 2001), Fribourg / Göttingen, p. 171-185.

PIRONTI Gabriella, 2005, « Aphrodite dans le domaine d'Arès. Éléments pour un dialogue entre mythe et culte ", à paraître dans Kernos 18 .

RUDHARDT Jean, 1981, Du mythe, de la religion et de la compréhension d'autrui, Genève.

--, 1990, « De la maternité chez les déesses grecques », Revue de l'Histoire des Religions, p. 367-388. SÉCHAN Louis, LÉVÊQUE Pierre, $1990^{2}$, Les grands dieux de la Grèce, Paris.

TRÜMPY Catherine, 2004, « Die Thesmophoria, Brimo, Deo und das Anaktoron : Beobachtungen zur Vorgeschichte des Demeterkults ", Kernos 17, p. 13-42.

\section{NOTES}

1. Séchan - Lévêque $1990^{2}$ [1966] : 136 ; Rudhardt $1990: 378$.

2. Rudhardt 1981 ; id. $1990: 378$.

3. C'est pourquoi l'Hymne homérique à Déméter a été associé tant aux Thesmophories qu'aux mystères d'Éleusis dont il raconte l'instauration. Parmi l'abondante bibliographie sur le thème, citons Foley (éd.) 1994 ; Calame 1997.

4. Cf. la mise au point récente de Trümpy 2004.

5. Dans une bibliographie immense, épinglons les travaux trop peu connus de Motte 1986a et 1986b.

6. Pausanias, V, 3,2 (trad. J. Pouilloux, CUF).

7. Pausanias, VI, 26, 3. 
8. Pausanias, V, 16, 6-7.

9. C'est le cas à Sparte dans un contexte similaire. Cf. Pirenne-Delforge 1994 : 204-205 ; Pironti 2005.

10. Loraux 1990 [1981] : 58-65. Le thème est également repris par Rudhardt 1990 :

382-383.

11. Euripide, Héraclides, 770-772. Mais elle n'est pas explicitement honorée comme telle. Sur un plan cultuel, la naissance et la maturation des enfants de l'Attique sont davantage prises en charge par une « entité » fonctionnelle appelée Kourotrophos (cf. Pirenne-Delforge 2004).

12. Pausanias, I, 27, 3.

13. Burkert 1966 ; Brulé 1987 : 79-139; Pirenne-Delforge 1994 : 50-59 ; Donnay 1997.

14. Rudhardt $1990: 383$.

15. Cf. Pausanias, V, 7, 6: «selon les Éléens qui rapportent les traditions les plus anciennes... »

16. Cf. par exemple Frazer 1969.

17. La dénonciation de ces généralisations abusives a commencé il y a plusieurs décennies : e.g. Georgoudi 1991 ; ead. 2002. - Cela n'a pas empêché de voir paraître, il y a moins de dix ans, une étude sacrifiant à nouveau aux pires des amalgames sous l'égide de M. Gimbutas à qui le volume est dédié : Haarmann 1996.

18. C'est ce que l'on appelle le « Goddess movement », sérieusement mis à mal dans l'ouvrage collectif publié par Goodison - Morris 1998.

\section{RÉSUMÉS}

Une première partie montre que, dans la Théogonie d'Hésiode, le terme de "mère " s'applique surtout à des déesses dont la relation à l'enfant est dramatisée au-delà de la seule référence généalogique. Une deuxième partie analyse les raisons de l'attribution du titre de "mère » à la déesse Athéna à Élis, par comparaison avec la situation d'Athènes. Une brève conclusion revient sur le fantasme d'une « Déesse-mère » générique en contexte grec.

The first part shows how, in the Hesiodic Theogony, the word « mother » is applied to goddesses who construct a specific attachment to their children, above and beyond the genealogical link. The second part considers why at Elis, Athena was called «mother ", comparing this situation with Athens. A brief conclusion comes back to the historiographical illusion of a generic « Mothergoddess » in the Greek world.

\section{AUTEUR}

\section{VINCIANE PIRENNE-DELFORGE}

Vinciane PIRENNE-DELFORGE est chercheuse au Fonds National de la Recherche Scientifique (Belgique) et maître de conférence à l'Université de Liège (cours d'Introduction à l'histoire des religions, de Pensée religieuse des Grecs et de Religion romaine). Son domaine de recherche est la religion grecque antique. Editrice de Kernos. Revue internationale et pluridisciplinaire de religion 
grecque (depuis 1988), elle a notamment publié : L'Aphrodite grecque, Athènes/Liège, 1994 (Kernos supplément 4) ; Mentor I (1992) et II (1998) : guides bibliographiques de la religion grecque antique ; (dir.), Les panthéons des cités grecques, des origines à la Périégèse de Pausanias, Liège,1998 (Kernos supplément 8) ; « Religion grecque », in Y. Lehmann (dir.), Religions de l'antiquité, Paris, PUF, 1999. 